\title{
The Contrast Mechanisms of LL-BSE Electrons in FE-SEM Characterization of Polymer, Single Proteins, and Oxidization States of Elements.
}

\author{
Dr. Heiner Jaksch \\ Carl Zeiss NTS GmbH, 73447 Oberkochen, Germany (jaksch@smt.zeiss.com)
}

The imaging of nano-material's and -compositions is of increasing importance and thus the role of detecting smallest compositional differences in materials becomes progressively more significant. Due to the high penetration of electrons in classical BSE imaging at high landing energies, this technology is unable to solve the demands of imaging nano-compositional materials with smallest compositional differences. Electrons penetrate and diffuse deeply into materials at high landing energies. The lower the density of the material, the higher is this penetration. This makes nano-particles and nano-layers "invisible" due to the large scattering volume. The demand is there to design a detection principle, capable to fulfill these demands.

Below a landing energies of $4 \mathrm{kV}$, the backscatter coefficient becomes non linear and drops with increasing atomic number stronger than that from elements with low atomic numbers (Fig:1a). At a certain landing energy we see equilibrium of backscatter yield and no contrast (Fig:1b). In the graph 1a one can see, that the element Chromium is at $1 \mathrm{kV}$ landing energy brighter than Gold. Carbon will be brighter than Gold around $400 \mathrm{eV}$ landing energy! Due to this fact and the problem, that the mean free path length of BSE electrons from low-density materials, such as proteins or polymers, becomes extremely small, we have introduced new technologies to visualize these low intensity signals coming from electrons with very small energy loss. The low loss BSE electrons are now introduced in SEM.

To understand the new contrast mechanisms experiments with hybrids, polymers and all kinds of different oxidization states of elements were made and will be shown. Essential for the contrast at low landing energies is not any more the atomic number or density as contrast mechanism, but only the bonding structure of the outer shell electrons or plasmon losses. To get the information from these electrons a double stage filtering is necessary. In the examples these results are explained with the hybridization of carbon as $\mathrm{sp}^{2}$ and $\mathrm{sp}^{3}$ hybrids and shown with imaging examples (Fig.2).

These hybrids are responsible for the contrast in all polymer and protein. In general we have to consider the bonding- / ionization energy or plasmon losses and not the nucleus charge as source of the contrast. In the shown examples it will be proven that density rules or z-number contrast fail in explaining the observed contrast. Monte Carlo simulations also are unable to model the fine contrast mechanisms.

The sensitivity of the technology is explained with the detection of a single protein $(8 \mathrm{~nm})$ in virus marked with GFP. The detection concept is verified with quantum dots (GaAlAs) of known band-gap with $2.5 \mathrm{eV}$ and $4.8 \mathrm{eV}$. What we see there is the resonance of a more or less free electron, replaced by the primary electrons. Such "free" electrons typically show extreme high contrast due to the very small energy loss when replaced by primary electrons. One can use this contrast mechanism to detect functional groups in polymers or as described above, in fluorophors in live science. As an outlook the technology will be a big step forward for the characterization of anything in live science and material science. 

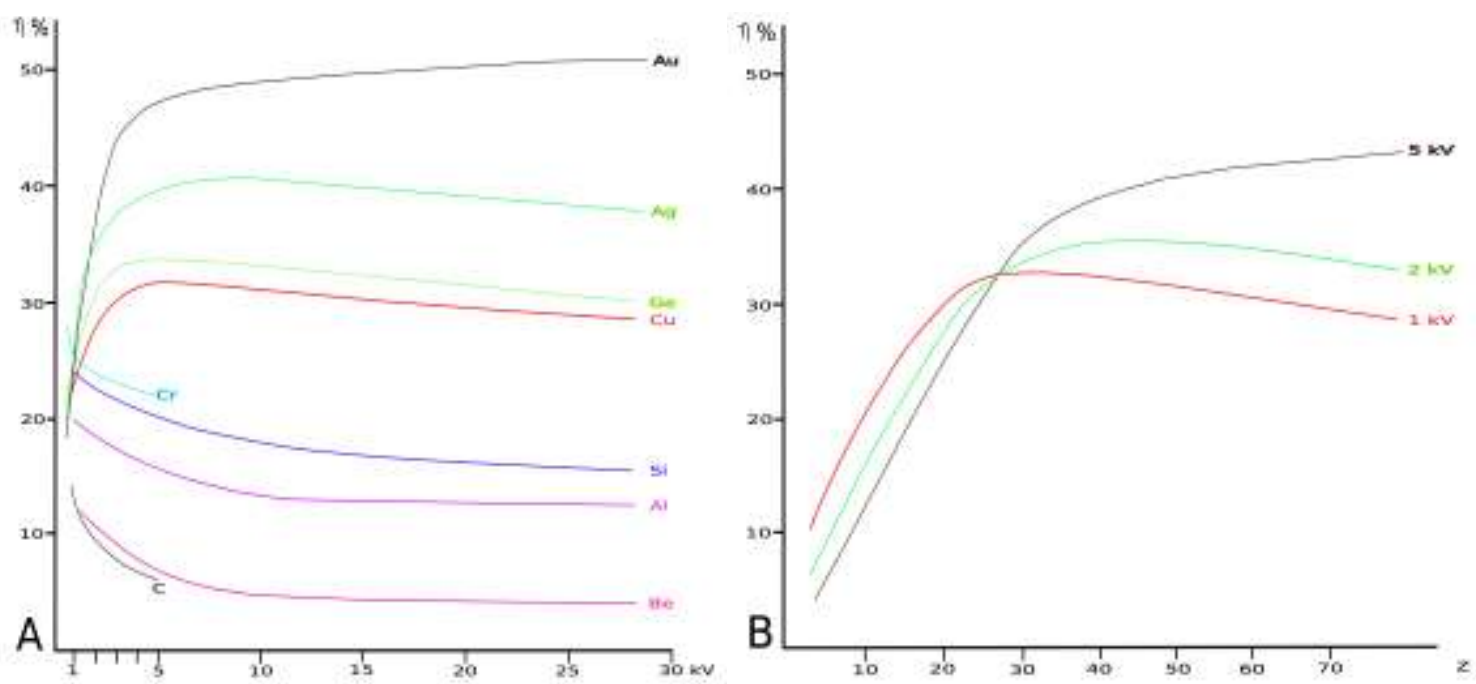

Fig.1:Backscatter coefficient as function of a) landing energy and as a function of b) atomic number Z (after:Reimer 1998).

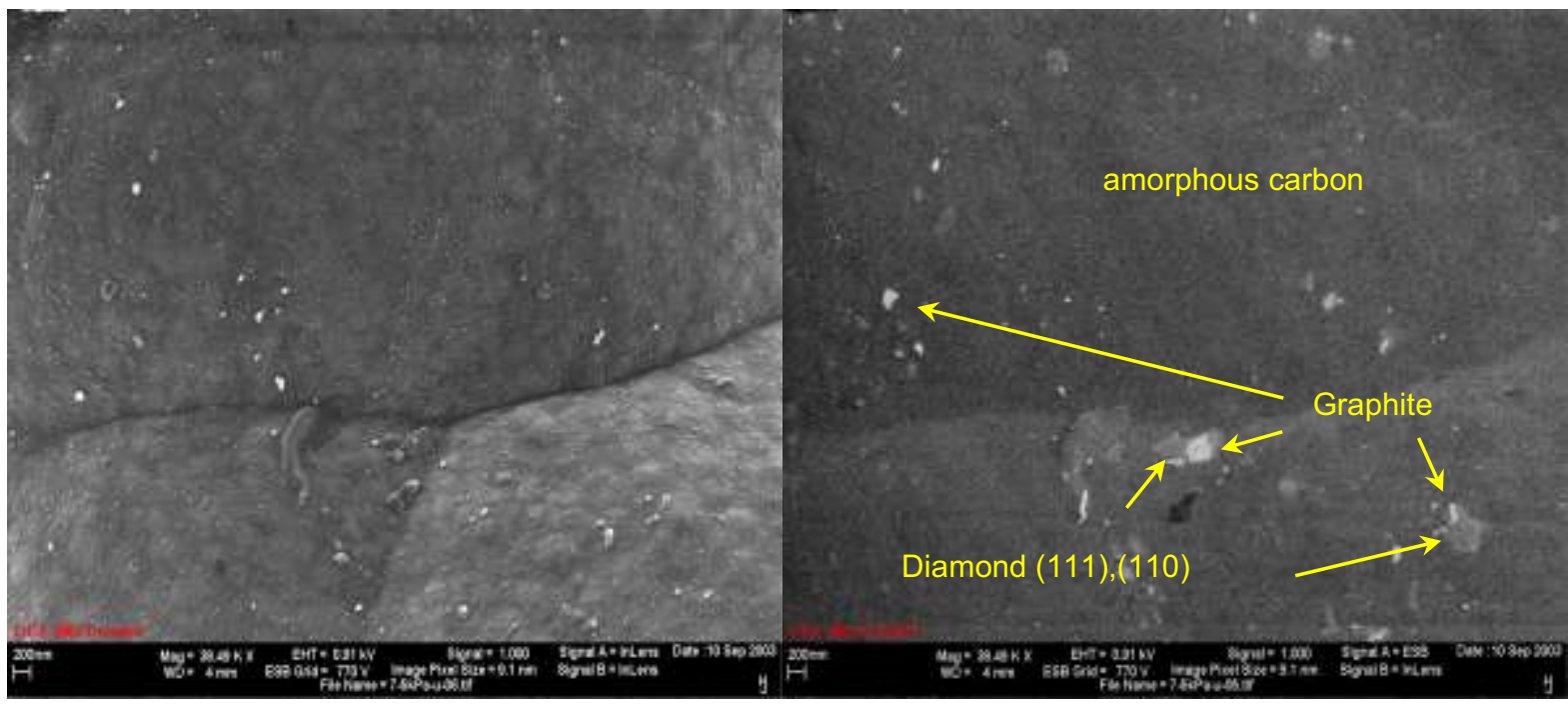

Fig. 2: SE electron image at 910 Volt landing energy of 3 modifications of carbon (left) and the corresponding LL-BSE image with $140 \mathrm{eV}$ low loss energy window, showing the $\mathrm{sp}^{2}-$ $\mathrm{sp}^{3}$ hybrids Diamond, Graphite and amorphous carbon. Note: Contrast inversion between Diamond and Graphite due to bonding energy,bonding type difference or plasmon losses!

\section{References}

[1] Jaksch H., Field emission SEM for true surface imaging and Analysis, Materials World, October 1996

[2] Jaksch H. et al. Microsc. Microanal.9 (Suppl) (2003) 106

[3] Jaksch H. et al. Microsc. Microanal.(2004) 1372 CD

[4] Reimer L., Scanning electron microscopy 2nd Edition, Springer-Verlag Berlin Heidelberg New York 1998

[5] Li H.M. and Ding Z.J., Monte Carlo Simulation of Secondary Electron and Backscatter Electron Images in Scanning Electron Microscopy for Specimen with Complex Geometry Structure

[6] Jaksch H., Low Loss BSE imaging in a FE-SEM, Proceedings IMC 2010, Rio de Janeiro 